\title{
Author Correction: Multicomponent electrocatalyst with ultralow Pt loading and high hydrogen evolution activity
}

Jitendra N. Tiwari, Siraj Sultan (D), Chang Woo Myung (D), Taeseung Yoon, Nannan Li, Miran Ha, Ahmad M. Harzandi, Hyo Ju Park, Dong Yeon Kim DD, S. Selva Chandrasekaran, Wang Geun Lee, Varun Vij, Hoju Kang, Tae Joo Shin, Hyeon Suk Shin (D), Geunsik Lee, Zonghoon Lee (iD) and Kwang S. Kim (D)

Correction to: Nature Energy https://doi.org/10.1038/s41560-018-0209-x, published online 30 July 2018.

In the version of this Article originally published, panel c was mistakenly omitted from Supplementary Fig. 6 . This has now been corrected.

Published online: 5 March 2019

https://doi.org/10.1038/s41560-019-0364-8 